Proceedings of the Edinburgh Mathematical Society (2003) 46, 229-249 (C)

DOI:10.1017/S0013091502000159 Printed in the United Kingdom

\title{
ON THE EXISTENCE OF MULTIPLE PERIODIC SOLUTIONS FOR EQUATIONS DRIVEN BY THE $p$-LAPLACIAN AND WITH A NON-SMOOTH POTENTIAL
}

\author{
LESZEK GASIŃSKI ${ }^{1}$ AND NIKOLAOS S. PAPAGEORGIOU ${ }^{2}$ \\ ${ }^{1}$ Jagiellonian University, Institute of Computer Science, \\ ul. Nawojki 11, 30072 Cracow, Poland \\ ${ }^{2}$ National Technical University, Department of Mathematics, \\ Zografou Campus, Athens 15780, Greece (npapg@math.ntua.gr)
}

(Received 11 February 2002)

\begin{abstract}
In this paper we examine periodic problems driven by the scalar $p$-Laplacian. Using nonsmooth critical-point theory and a recent multiplicity result based on local linking (the original smooth version is due to Brezis and Nirenberg), we prove three multiplicity results, the third for semilinear problems with resonance at zero. We also study a quasilinear periodic eigenvalue problem with the parameter near resonance. We prove the existence of three distinct solutions, extending in this way a semilinear and smooth result of Mawhin and Schmitt.
\end{abstract}

Keywords: non-smooth critical-point theory; locally Lipschitz functional; Clarke subdifferential; non-smooth Palais-Smale condition; local linking; coercive functional

AMS 2000 Mathematics subject classification: Primary 34C25

\section{Introduction}

Recently, there has been increasing interest in scalar periodic problems driven by the onedimensional $p$-Laplacian. We refer to the works of Del Pino, Manasevich and Murua [7], Fabry and Fayyad [8], Guo [10] and Dang and Oppenheimer [6]. In all these works the approach is degree theoretical and they establish the existence of one solution. Only Del Pino, Manasevich and Murua [7] examine the problem of existence of multiple periodic solutions. Their approach uses degree theory and assumes that the right-hand side nonlinearity $f(t, \zeta)$ is jointly continuous in $t \in T=[0, b]$ and $\zeta \in \mathbb{R}$. Their conditions on $f$ also require that asymptotically there is no interaction between the nonlinearity and the Fučik spectrum of the one-dimensional $p$-Laplacian. The study of problems with nonsmooth potential is lagging behind: there are only the works of Adly and Goeleven [2] (semilinear problems, i.e. $p=2$ ) and of Gasiński and Papageorgiou [9] (problems with the $p$-Laplacian). In both of these papers the approach is variational and is based on the non-smooth critical-point theory of Chang [4] and its extensions due to Kourogenis and Papageorgiou [13]. In Gasiński and Papageorgiou [9] the authors prove the existence of three distinct periodic solutions. 
In this paper, for this problem, we prove three multiplicity theorems, the third concerning semilinear problems (i.e. $p=2$ ). These results use a recent non-smooth extensiondue to Kourogenis, Kandilakis and Papageorgiou [14] - of a local linking theorem of Brezis and Nirenberg [3]. In the last section we consider a nonlinear periodic eigenvalue problem driven by the $p$-Laplacian and for $\lambda$ approaching zero from below, we show that the problem has at least three distinct solutions. Our result partly extends Theorem 4 of Mawhin and Schmitt [17], where the problem is semilinear (i.e. $p=2$ ) and the potential function is $C^{1}$.

The problem under consideration is the following:

$$
\begin{gathered}
-\left(\left|x^{\prime}(t)\right|^{p-2} x^{\prime}(t)\right)^{\prime} \in \partial j(t, x(t)) \quad \text { for almost all } t \in T=[0, b], \\
x(0)=x(b), \quad x^{\prime}(0)=x^{\prime}(b), \quad 1<p<\infty .
\end{gathered}
$$

Here $\partial j(t, \cdot)$ stands for the Clarke subdifferential of the locally Lipschitz (not necessarily smooth) potential function $j(t, \cdot)$.

\section{Mathematical background}

As we have already mentioned our approach will be variational and will be based on the non-smooth critical-point theory of Chang [4]. The theory of Chang [4] uses the subdifferential theory of Clarke [5] for locally Lipschitz functions. For the convenience of the reader we present below the main aspects of this theory that we shall need. Details can be found in the book by Clarke [5].

Let $X$ be a Banach space and $X^{*}$ its topological dual. By $\langle\cdot, \cdot\rangle_{X}$ we denote the duality brackets for the pair $\left(X, X^{*}\right)$. A function $\varphi: X \mapsto \mathbb{R}$ is said to be locally Lipschitz if, for every bounded open set $U \subseteq X$, we can find $k_{U}>0$ such that $|\varphi(x)-\varphi(y)| \leqslant k_{U}\|x-y\|_{X}$ for all $x, y \in U$. Recall that if $\psi: X \rightarrow \overline{\mathbb{R}} \stackrel{\text { df }}{=} \mathbb{R} \cup\{+\infty\}$ is proper (i.e. $\operatorname{dom} \psi \stackrel{\mathrm{df}}{=}\{x \in X: \psi(x)<+\infty\} \neq \emptyset$ ), convex and lower semicontinuous (i.e. $\psi \in \Gamma_{0}(X)$, see Hu and Papageorgiou [11, p. 341]), then $\psi$ is locally Lipschitz in the interior of its effective domain $\operatorname{dom} \psi$. So if $X$ is finite dimensional, a convex $\mathbb{R}$-valued function is locally Lipschitz.

Given a locally Lipschitz function $\varphi: X \rightarrow \mathbb{R}$, we define the generalized directional derivative $\varphi^{0}(x ; h)$ of $\varphi$ at $x \in X$ in the direction $h \in X$, by

$$
\varphi^{0}(x ; h) \stackrel{\text { df }}{=} \limsup _{\substack{y \rightarrow x \\ t \searrow 0}} \frac{\varphi(y+t h)-\varphi(y)}{t} .
$$

It is easy to see that $X \ni h \mapsto \varphi^{0}(x ; h) \in \mathbb{R}$ is sublinear, continuous and so by the HahnBanach Theorem, $\varphi^{0}(x ; \cdot)$ is the support function of a non-empty, weak ${ }^{*}$-compact and convex set $\partial \varphi(x)$ defined by

$$
\partial \varphi(x) \stackrel{\text { df }}{=}\left\{x^{*} \in X^{*}:\left\langle x^{*}, h\right\rangle_{X} \leqslant \varphi^{0}(x ; h) \forall h \in X\right\} .
$$

The multifunction $\partial \varphi: X \rightarrow 2^{X^{*}} \backslash\{\emptyset\}$ is known as the Clarke or generalized subdifferential of $\varphi$. This multifunction has a graph

$$
\operatorname{Gr} \partial \varphi \stackrel{\text { df }}{=}\left\{\left(x, x^{*}\right) \in X \times X^{*}: x^{*} \in \partial \varphi(x)\right\},
$$


which is sequentially closed in $X \times X_{\mathrm{w}^{*}}^{*}$. Here by $X_{\mathrm{w}^{*}}^{*}$ we denote the Banach space $X^{*}$ furnished with the weak ${ }^{*}$-topology. So if $x_{n} \rightarrow x$ in $X, x_{n}^{*} \rightarrow x^{*}$ weakly* in $X^{*}$ and $x_{n}^{*} \in \partial \varphi\left(x_{n}\right)$ for all $n \geqslant 1$, then $x^{*} \in \partial \varphi(x)$. If $\varphi, \psi: X \rightarrow \mathbb{R}$ are both locally Lipschitz functionals, then $\partial(\varphi+\psi)(x) \subseteq \partial \varphi(x)+\partial \psi(x)$ and $\partial(t \varphi)(x)=t \varphi(x)$ for all $x \in X$ and all $t \in \mathbb{R}$. If in addition $\varphi$ is convex, then the subdifferential $\partial \varphi$ coincides with the subdifferential in the sense of convex analysis (see Hu and Papageorgiou [11, p. 267]). Finally, if $\varphi$ is continuously Gateaux differentiable at $x \in X$ (i.e. $\varphi \in C^{1}(X)$ ), then $\partial \varphi(x)=\left\{\varphi^{\prime}(x)\right\}$.

Let $\varphi: X \rightarrow \mathbb{R}$ be a locally Lipschitz function. We say that $x \in X$ is a critical point of $\varphi$, if $0 \in \partial \varphi(x)$. Then $c \stackrel{d f}{=} \varphi(x)$ is a critical value of $\varphi$. It is easy to check that if $x \in X$ is a local extremum (i.e. local maximum or minimum), then $x$ is a critical point, i.e. $0 \in \partial \varphi(x)$. It is well known that the smooth critical-point theory uses a compactness condition, known as the Palais-Smale condition. In the present non-smooth setting, this condition takes the following form.

A locally Lipschitz function $\varphi: X \rightarrow \mathbb{R}$ satisfies the non-smooth Palais-Smale condition, if any sequence $\left\{x_{n}\right\}_{n \geqslant 1} \subseteq X$ such that $\left\{\varphi\left(x_{n}\right)\right\}_{n \geqslant 1}$ is bounded and $m\left(x_{n}\right) \rightarrow 0$ as $n \rightarrow+\infty$ (where $\left.m\left(x_{n}\right) \stackrel{\text { df }}{=} \inf \left\{\left\|x^{*}\right\|_{*}: x^{*} \in \partial \varphi\left(x_{n}\right)\right\}\right)$ has a strongly convergent subsequence.

If $\varphi \in C^{1}(X)$, then because $\partial \varphi(x)=\left\{\varphi^{\prime}(x)\right\}$ for all $x \in X$, we see that the notion of the non-smooth Palais-Smale condition coincides with the classical one (see Mawhin and Willem [18, p. 130]). Using this condition, Chang [4] proved a deformation theorem and then obtained a non-smooth version of the classical Saddle Point Theorem (for a more general version see Kourogenis and Papageorgiou [13]).

Theorem 2.1. If

(a) $X$ is a reflexive Banach space, $X=Y \oplus V$ with $\operatorname{dim} Y<+\infty$;

(b) $\varphi: X \rightarrow \mathbb{R}$ is a locally Lipschitz functional;

(c) there exists $R>0$ such that

$$
\max \left\{\varphi(y): y \in Y,\|y\|_{X}=R\right\}<\inf \{\varphi(v): v \in V\} ;
$$

(d) $\varphi$ satisfies the non-smooth Palais-Smale condition;

(e) $c_{0} \stackrel{\mathrm{df}}{=} \inf _{\gamma \in \Gamma} \max _{y \in D} \varphi(\gamma(y))$, with $D \stackrel{\mathrm{df}}{=}\left\{y \in Y:\|y\|_{X} \leqslant R\right\}$ and

$$
\Gamma \stackrel{\mathrm{df}}{=}\left\{\gamma \in C(D ; X): \gamma(y)=y \text { for }\|y\|_{X}=R\right\},
$$

then $c_{0} \geqslant \inf _{v \in V} \varphi(v)$ and $c_{0}$ is a critical value of $\varphi$.

Moreover, if $c_{0}=\inf _{v \in V} \varphi(v)$, then there exists a critical point $x \in V$ of $\varphi$ with $c_{0}=\varphi(x)$. 
The next result is a non-smooth generalization of the local linking theorem of Brezis and Nirenberg [3] and it is due to Kourogenis, Kandilakis and Papageorgiou [14].

Theorem 2.2. If

(a) $X$ is a reflexive Banach space, $X=Y \oplus V$ with $\operatorname{dim} Y<+\infty$;

(b) $\varphi: X \rightarrow \mathbb{R}$ is a locally Lipschitz functional which is bounded below;

(c) $\varphi$ satisfies the non-smooth Palais-Smale condition;

(d) $\varphi(0)=0$ and $\inf _{x \in X} \varphi(x)<0$;

(e) there exists $r>0$ such that

$$
\begin{array}{ll}
\varphi(x) \leqslant 0 & \text { if } x \in Y \text { and }\|x\|_{X} \leqslant r \\
\varphi(x) \geqslant 0 & \text { if } x \in V \text { and }\|x\|_{X} \leqslant r
\end{array}
$$

then $\varphi$ has at least two non-trivial critical points.

Remark 2.3. Actually the result of Kourogenis, Kandilakis and Papageorgiou [14] is more general than the above theorem, since it assumes that $\varphi$ satisfies the non-smooth Cerami condition (see Kourogenis, Kandilakis and Papageorgiou [14]). Even when specialized to the non-smooth setting (i.e. $\varphi \in C^{1}(X)$ ), this is a slight improvement of the original result of Brezis and Nirenberg [3].

By an eigenvalue $\lambda$ of the minus scalar $p$-Laplacian $x \mapsto-\left(\left|x^{\prime}\right|^{p-2} x^{\prime}\right)^{\prime}$ with $b$-periodic boundary condition, we mean a $\lambda \in \mathbb{R}$ such that the problem

$$
\left.\begin{array}{c}
-\left(\left|x^{\prime}(t)\right|^{p-2} x^{\prime}(t)\right)^{\prime}=\lambda|x(t)|^{p-2} x(t) \quad \text { for almost all } t \in T=[0, b], \\
x(0)=x(b), \quad x^{\prime}(0)=x^{\prime}(b),
\end{array}\right\}
$$

has a non-trivial solution $x$, called the eigenfunction corresponding to $\lambda$. Let $S(p)$ be the set of these eigenvalues. Evidently, $0 \in S(p)$, i.e. $S(p) \neq \emptyset$. A direct integration of (EP) gives that $S(p)$ consists of the sequence

$$
\lambda_{n}(p)=\left(\frac{2 n \pi_{p}}{b}\right)^{p}=\frac{(p-1)(n \omega)^{p}}{(p \sin (\pi / p))^{p}}
$$

where

$$
\omega \stackrel{\mathrm{df}}{=} \frac{2 \pi}{b} \quad \text { and } \quad \pi_{p} \stackrel{\mathrm{df}}{=} 2(p-1)^{1 / p} \int_{0}^{1}\left(1-t^{p}\right)^{-1 / p}=2(p-1)^{1 / p} \frac{\pi / p}{p \sin (\pi / p)} .
$$

Each eigenvalue is non-negative and 0 is the smallest one (see Mawhin [16]). In what follows we will need the following function space:

$$
W_{\text {per }}^{1, p}(T) \stackrel{\text { df }}{=}\left\{x \in W^{1, p}(T): x(0)=x(b)\right\} .
$$




\section{Multiplicity results}

The problem under consideration is

$$
\left.\begin{array}{c}
-\left(\left|x^{\prime}(t)\right|^{p-2} x^{\prime}(t)\right)^{\prime} \in \partial j(t, x(t)) \quad \text { for almost all } t \in T=[0, b], \\
x(0)=x(b), \quad x^{\prime}(0)=x^{\prime}(b),
\end{array}\right\}
$$

where $1<p<\infty$. Here by $j(t, \zeta)$ we denote a function which is locally Lipschitz in the $\zeta \in \mathbb{R}$-variable and $\partial j(t, \zeta)$ is the subdifferential in the sense of Clarke (see $\S 2$ ). If $j(t, \zeta)=\int_{0}^{\zeta} f(t, r) \mathrm{d} r$ and $f(t, \cdot)$ is continuous, then $j(t, \cdot) \in C^{1}(\mathbb{R})$ and $\partial j(t, \zeta)=\left\{j_{\zeta}^{\prime}(t, \zeta)\right\}$.

For the first multiplicity result our assumptions on the non-smooth potential $j(t, \zeta)$ are as follows.

$\left(H(j)_{1}\right) \cdot j: T \times \mathbb{R} \mapsto \mathbb{R}$ is a functional such that

(i) for all $\zeta \in \mathbb{R}$, the function $T \ni t \mapsto j(t, \zeta) \in \mathbb{R}$ is measurable;

(ii) for almost all $t \in T$, the function $\mathbb{R} \ni \zeta \mapsto j(t, \zeta) \in \mathbb{R}$ is locally Lipschitz and $j(t, 0)=0$

(iii) for almost all $t \in T$, all $\zeta \in \mathbb{R}$ and all $u \in \partial j(t, \zeta)$ we have

$$
|u| \leqslant a(t)+c(t)|\zeta|^{r-1},
$$

with $a, c \in L^{r^{\prime}}(T)_{+}$, where $1 \leqslant r<+\infty$ and $(1 / r)+\left(1 / r^{\prime}\right)=1$;

(iv) $\lim \sup _{|\zeta| \rightarrow+\infty}\left(p j(t, \zeta) /|\zeta|^{p}\right)<0$ uniformly for almost all $t \in T$; and

(v) there exist $\rho_{1}>0$ and $0<\mu<\left(1 / b^{p}\right)$, such that for almost all $t \in T$ and all $|\zeta| \leqslant \rho_{1}$ we have $0 \leqslant p j(t, \zeta) \leqslant \mu|\zeta|^{p}$.

Theorem 3.1. If hypotheses $H(j)_{1}$ hold, then problem (HVI) has at least two distinct non-trivial solutions.

Proof. By virtue of hypothesis $H(j)_{1}$ (iv), we can find $\beta>0$ and $M_{1} \geqslant 1$ such that for almost all $t \in T$ and all $|\zeta| \geqslant M_{1}$ we have

$$
j(t, \zeta) \leqslant-(\beta / p)|\zeta|^{p}
$$

On the other hand, from the mean value theorem of Lebourg [15] (see also Clarke [5, p. 41]) and hypotheses $H(j)_{1}$ (ii), (iii), for almost all $t \in T$ and all $\zeta \in \mathbb{R}$ we have that

$$
|j(t, \zeta)| \leqslant a_{1}(t)+c_{1}(t)|\zeta|^{r}
$$

with $a_{1} \in L^{1}(T)_{+}, c_{1} \in L^{r^{\prime}}(T)_{+}$. So for almost all $t \in T$ and all $|\zeta|<M_{1}$ we have

$$
|j(t, \zeta)| \leqslant a_{2}(t)
$$


with $a_{2} \in L^{1}(T)$. Thus, finally, for almost all $t \in T$ and all $\zeta \in \mathbb{R}$, we can write that

$$
j(t, \zeta) \leqslant-(\beta / p)|\zeta|^{p}+a_{3}(t)
$$

with $a_{3} \in L^{1}(T)$. Let $\varphi \in W_{\text {per }}^{1, p}(T) \rightarrow \mathbb{R}$ be defined by

$$
\varphi(x) \stackrel{\mathrm{df}}{=} \frac{1}{p}\left\|x^{\prime}\right\|_{p}^{p}-\int_{0}^{b} j(t, x(t)) \mathrm{d} t .
$$

We know that $\varphi$ is locally Lipschitz (see Chang [4] or Hu and Papageorgiou [12, p. 313]). Also, for $x \in W_{\text {per }}^{1, p}(T)$, we know that

$$
\begin{aligned}
\varphi(x) & =\frac{1}{p}\left\|x^{\prime}\right\|_{p}^{p}-\int_{0}^{b} j(t, x(t)) \mathrm{d} t \geqslant \frac{1}{p}\left\|x^{\prime}\right\|_{p}^{p}+\frac{\beta}{p}\|x\|_{p}^{p}-\beta_{1} \\
& \geqslant \frac{1}{p} \min \{1, \beta\}\left(\left\|x^{\prime}\right\|_{p}^{p}+\|x\|_{p}^{p}\right)-\beta_{1}
\end{aligned}
$$

with $\beta_{1}>0$. From this inequality it follows that $\varphi$ is coercive. Therefore, $\varphi$ is bounded below. Also it satisfies the non-smooth Palais-Smale condition. Indeed let $\left\{x_{n}\right\}_{n \geqslant 1} \subseteq W_{\text {per }}^{1, p}(T)$ be a sequence such that $\left|\varphi\left(x_{n}\right)\right| \leqslant M_{2}$ for all $n \geqslant 1$ with some $M_{2}>0$ and $m\left(x_{n}\right) \rightarrow 0$. Since $\varphi$ is coercive and the sequence $\left\{\varphi\left(x_{n}\right)\right\}_{n \geqslant 1}$ is bounded, it also follows that the sequence $\left\{x_{n}\right\}_{n} \geqslant 1 \subseteq W_{\text {per }}^{1, p}(T)$ is bounded and so, by passing to a subsequence if necessary, we may assume that $x_{n} \rightarrow x$ weakly in $W_{\text {per }}^{1, p}(T)$ and $x_{n} \rightarrow x$ in $C(T)$ (from the compactness of the embedding $W_{\text {per }}^{1, p}(T) \subseteq C(T)$ ). Also let $x_{n}^{*} \in \partial \varphi\left(x_{n}\right)$, $n \geqslant 1$, be such that $m\left(x_{n}\right)=\left\|x_{n}^{*}\right\|_{*}$. The existence of such elements follows from the weak compactness of sets $\partial \varphi\left(x_{n}\right) \subseteq W_{\text {per }}^{1, p}(T)$ and the weak lower semicontinuity of the norm functional in Banach space. We have

$$
x_{n}^{*}=A\left(x_{n}\right)-u_{n},
$$

with $A: W_{\text {per }}^{1, p}(T) \rightarrow W_{\text {per }}^{1, p}(T)^{*}$ being the operator defined by

$$
\langle A(x), y\rangle=\int_{0}^{b}\left|x^{\prime}(t)\right|^{p-2} x^{\prime}(t) y^{\prime}(t) \mathrm{d} t \quad \forall x, y \in W_{\text {per }}^{1, p}(T)
$$

and

$$
u_{n} \in S_{\partial j\left(\cdot, x_{n}(\cdot)\right)}^{r^{\prime}} \stackrel{\text { df }}{=}\left\{v \in L^{r^{\prime}}(T): v(t) \in \partial j\left(t, x_{n}(t)\right) \text { a.e. on } T\right\} .
$$

We know that $A$ is monotone, demicontinuous, hence maximal monotone (see Hu and Papageorgiou [11, p. 309]). From the choice of the sequence $\left\{x_{n}\right\}_{n \geqslant 1} \subseteq W_{\text {per }}^{1, p}(T)$, we have that

$$
\left\langle A\left(x_{n}\right), x_{n}-x\right\rangle-\int_{0}^{b} u_{n}(t)\left(x_{n}-x\right)(t) \mathrm{d} t \leqslant \varepsilon_{n}\left\|x_{n}-x\right\|_{1, p}
$$

and so

$$
\limsup _{n \rightarrow+\infty}\left\langle A\left(x_{n}\right), x_{n}-x\right\rangle \leqslant 0 .
$$

But $A$, being maximal monotone, is generalized pseudomonotone (see $\mathrm{Hu}$ and Papageorgiou [11, p. 365]) and so $\left\langle A\left(x_{n}\right), x_{n}\right\rangle \rightarrow\langle A(x), x\rangle$, hence $\left\|x_{n}^{\prime}\right\|_{p} \rightarrow\left\|x^{\prime}\right\|_{p}$. Since $x_{n}^{\prime} \rightarrow x^{\prime}$ 
weakly in $L^{p}(T)$ and $L^{p}(T)$ is uniformly convex, it follows that $x_{n}^{\prime} \rightarrow x^{\prime}$ in $L^{p}(T)$ (the Kadec-Klee property) and so $x_{n} \rightarrow x$ in $W_{\text {per }}^{1, p}(T)$. This proves that $\varphi$ satisfies the nonsmooth Palais-Smale condition.

Next we consider the direct sum decomposition

$$
W_{\text {per }}^{1, p}(T)=\mathbb{R} \oplus V \quad \text { with } V \stackrel{\text { df }}{=}\left\{v \in W_{\text {per }}^{1, p}(T): \int_{0}^{b} v(t) \mathrm{d} t=0\right\} .
$$

First let $\eta \in \mathbb{R}$ be such that $|\eta| \leqslant \rho_{1}$. Then from hypothesis $H(j)_{1}(\mathrm{v})$ we have that

$$
\varphi(\eta)=-\int_{0}^{b} j(t, \eta) \mathrm{d} t \leqslant 0 .
$$

Also, if $v \in V$, because of the compactness of the embedding $W_{\text {per }}^{1, p}(T) \subseteq C(T)$, we can find $0<\rho_{2} \leqslant \rho_{1}$ such that if $\|v\|_{1, p} \leqslant \rho_{2}$, then $|v(t)| \leqslant \rho_{1}$ for all $t \in T$. So, from hypothesis $H(j)_{1}(\mathrm{v})$, for every $v \in V$ with $\|v\|_{1, p} \leqslant \rho_{2}$ we have that

$$
\begin{aligned}
\varphi(v) & =\frac{1}{p}\left\|v^{\prime}\right\|_{p}^{p}-\int_{0}^{b} j(t, v(t)) \mathrm{d} t \\
& =\frac{1}{p}\left\|v^{\prime}\right\|_{p}^{p}-\int_{\left\{|v| \leqslant \rho_{1}\right\}} j(t, v(t)) \mathrm{d} t \geqslant \frac{1}{p}\left\|v^{\prime}\right\|_{p}^{p}-\frac{\mu}{p}\|v\|_{p}^{p} .
\end{aligned}
$$

But from the Poincaré-Wirtinger inequality (see Mawhin and Willem [18, p. 8]) we have that

$$
\|v\|_{p}^{p} \leqslant b\|v\|_{\infty}^{p} \leqslant b b^{p-1}\left\|v^{\prime}\right\|_{p}^{p}=b^{p}\left\|v^{\prime}\right\|_{p}^{p} \quad \forall v \in V .
$$

Recalling the hypothesis on $\mu$ (see hypothesis $H(j)_{1}(\mathrm{v})$ ), for all $v \in V$ with $\|v\|_{1, p} \leqslant \rho_{2}$ we have that

$$
\varphi(v) \geqslant \frac{1}{p}\left\|v^{\prime}\right\|_{p}^{p}-\frac{\mu}{p} b^{p}\left\|v^{\prime}\right\|_{p}^{p}=\frac{1}{p}\left(1-\mu b^{p}\right)\left\|v^{\prime}\right\|_{p}^{p} \geqslant 0 .
$$

Finally, note that by virtue of hypothesis $H(j)_{1}$ (iv) we have that

$$
\inf \left\{\varphi(x): x \in W_{\text {per }}^{1, p}(T)\right\}<0 .
$$

Therefore, we can apply Theorem 2.2 and produce two distinct non-trivial critical points $\bar{x}, \overline{\bar{x}} \in W_{\text {per }}^{1, p}(T)$ of $\varphi$. We have $0 \in \partial \varphi(\bar{x})$ and so we can find $\bar{u} \in S_{\partial j(\cdot, \bar{x}(\cdot))}^{r^{\prime}}$ such that

$$
A(\bar{x})=\bar{u} .
$$

For all $\vartheta \in C_{0}^{\infty}(T)$ we have

$$
\langle A(\bar{x}), \vartheta\rangle=\int_{0}^{b} \bar{u}(t) \vartheta(t) \mathrm{d} t
$$

and so

$$
\int_{0}^{b}\left|\bar{x}^{\prime}(t)\right|^{p-2} \bar{x}^{\prime}(t) \vartheta^{\prime}(t) \mathrm{d} t=\int_{0}^{b} \bar{u}(t) \vartheta(t) \mathrm{d} t .
$$


Note that $\left(\left|\bar{x}^{\prime}\right|^{p-2} \bar{x}^{\prime}\right)^{\prime} \in W^{-1, p^{\prime}}(T)=\left(W_{0}^{1, p}(T)\right)^{*}$, where $(1 / p)+\left(1 / p^{\prime}\right)=1$ (see, for example, Adams [1, p. 50]). So we obtain

$$
\left\langle-\left(\left|\bar{x}^{\prime}\right|^{p-2} \bar{x}^{\prime}\right)^{\prime}, \vartheta\right\rangle_{0}=\langle\bar{x}, \vartheta\rangle_{0}
$$

where by $\langle\cdot, \cdot\rangle_{0}$ we denote the duality brackets for the following pair of spaces $\left(W_{0}^{1, p}(T), W^{-1, p^{\prime}}(T)\right)$. Recall that $C_{0}^{\infty}(T)$ is dense in $W_{0}^{1, p}(T)$. So we obtain

$$
-\left(\left|\bar{x}^{\prime}(t)\right|^{p-2} \bar{x}^{\prime}(t)\right)^{\prime}=\bar{u}(t) \in \partial j(t, \bar{x}(t)) \quad \text { a.e. on } T \text {. }
$$

Hence $\left|\bar{x}^{\prime}(\cdot)\right|^{p-2} \bar{x}^{\prime}(\cdot) \in W^{1, p^{\prime}}(T) \subseteq C(T)$. From Green's identity, for every $w \in W_{\text {per }}^{1, p}(T)$ we have

$$
\langle A(\bar{x}), w\rangle+\left\langle\left(\left|\bar{x}^{\prime}\right|^{p-2} \bar{x}^{\prime}\right)^{\prime}, w\right\rangle=\left|\bar{x}^{\prime}(b)\right|^{p-2} \bar{x}^{\prime}(b) w(b)-\left|\bar{x}^{\prime}(0)\right|^{p-2} \bar{x}^{\prime}(0) w(0),
$$

so from (3.1) and (3.2) we have that

$$
\left|\bar{x}^{\prime}(0)\right|^{p-2} \bar{x}^{\prime}(0) w(0)=\left|\bar{x}^{\prime}(b)\right|^{p-2} \bar{x}^{\prime}(b) w(b) \quad \forall w \in W_{\mathrm{per}}^{1, p}(T) .
$$

Let $w \in W_{\text {per }}^{1, p}(T)$ be such that $w(0)=w(b)=1$. We obtain

$$
\left|\bar{x}^{\prime}(0)\right|^{p-2} \bar{x}^{\prime}(0)=\left|\bar{x}^{\prime}(b)\right|^{p-2} \bar{x}^{\prime}(b)
$$

and so

$$
\bar{x}^{\prime}(0)=\bar{x}^{\prime}(b)
$$

i.e. $\bar{x}$ is a solution of (HVI).

Similarly, since $0 \in \partial \varphi(\overline{\bar{x}})$ we have that $\overline{\bar{x}} \in W_{\text {per }}^{1, p}(T)$ is another non-trivial solution of (HVI). Therefore, (HVI) has at least two distinct non-trivial solutions.

In the previous theorem, hypothesis $H(j)_{1}$ (iv) implies that at $\pm \infty$ there is no interaction of the non-smooth potential $j$ with the spectrum of minus the periodic scalar $p$ Laplacian (see $\S 2$ ). In the next multiplicity result we allow such interaction (resonance) at $\pm \infty$. More precisely, our hypotheses on the potential $j(t, \zeta)$ are as follows.

$\left(H(j)_{2}\right) \cdot j: T \times \mathbb{R} \mapsto \mathbb{R}$ is a functional such that

(i) for all $\zeta \in \mathbb{R}$, the function $T \ni t \mapsto j(t, \zeta) \in \mathbb{R}$ is measurable;

(ii) for almost all $t \in T$, the function $\mathbb{R} \ni \zeta \mapsto j(t, \zeta) \in \mathbb{R}$ is locally Lipschitz and $j(t, 0)=0$;

(iii) for almost all $t \in T$, all $\zeta \in \mathbb{R}$ and all $u \in \partial j(t, \zeta)$ we have

$$
|u| \leqslant a(t)+c(t)|\zeta|^{r-1}
$$

with $a, c \in L^{r^{\prime}}(T)$, where $1 \leqslant r<+\infty$ and $(1 / r)+\left(1 / r^{\prime}\right)=1$;

(iv) $\lim _{|\zeta| \rightarrow+\infty}\left(p j(t, \zeta) /|\zeta|^{p}\right)=0$ uniformly for almost all $t \in T$; 
(v) $\lim _{|\zeta| \rightarrow+\infty}(\zeta u-p j(t, \zeta))=+\infty$ uniformly for almost all $t \in T$ and all $u \in \partial j(t, \zeta)$; and

(vi) there exist $\rho_{1}>0$ and $0<\mu<\left(1 / b^{p}\right)$, such that for almost all $t \in T$ and all $|\zeta| \leqslant \rho_{1}$ we have $0 \leqslant p j(t, \zeta) \leqslant \mu|\zeta|^{p}$.

Remark 3.2. Consider the potential function

$$
j(t, \zeta) \stackrel{\text { df }}{=} \begin{cases}\frac{\mu}{p} \sin |\zeta|^{p} & \text { if }|\zeta| \leqslant 1, \\ \ln |\zeta|-\frac{a(t)}{r}|\zeta|^{r}+\frac{\mu}{p} \sin 1+\frac{a(t)}{r} & \text { if }|\zeta|>1 .\end{cases}
$$

If $\mu<\left(1 / b^{p}\right)$ and $a \in L^{r^{\prime}}(T)_{+}$with $(1 / r)+\left(1 / r^{\prime}\right)=1$ and $1 \leqslant r<p$, then hypotheses $H(j)_{2}$ are satisfied with $\rho_{1}=1$.

Theorem 3.3. If hypotheses $H(j)_{2}$ hold, then problem (HVI) has at least two distinct non-trivial solutions.

Proof. By virtue of hypothesis $H(j)_{2}$ (v) for a given $\beta>0$ we can find $M_{\beta}>0$ such that for almost all $t \in T$ and all $|\zeta| \geqslant M_{\beta}$ and all $u \in \partial j(t, \zeta)$ we have

$$
\zeta u-p j(t, \zeta) \geqslant \beta .
$$

From Clarke [5, p. 48], we know that for almost all $t \in T$ and all $\zeta>0$, the function $\zeta \mapsto\left(j(t, \zeta) / \zeta^{p}\right)$ is locally Lipschitz and we have

$$
\begin{aligned}
\partial\left(\frac{j(t, \zeta)}{\zeta^{p}}\right) & =\frac{\zeta^{p} \partial j(t, \zeta)-p \zeta^{p-2} \zeta j(t, \zeta)}{\zeta^{2 p}} \\
& =\zeta^{p-1}\left(\frac{\zeta \partial j(t, \zeta)-p j(t, \zeta)}{\zeta^{2 p}}\right) \\
& =\frac{\zeta \partial j(t, \zeta)-p j(t, \zeta)}{\zeta^{p+1}}
\end{aligned}
$$

So, from (3.3), for almost all $t \in T$, all $\zeta \geqslant M_{\beta}$ and all $v \in \partial\left(j(t, \zeta) / \zeta^{p}\right)$ we have

$$
v \geqslant \frac{\beta}{\zeta^{p+1}}
$$

Since for $t \in T \backslash E$, with $|E|=0$, the function $\zeta \mapsto\left(j(t, \zeta) / \zeta^{p}\right)$ is locally Lipschitz on $\left[M_{\beta},+\infty\right)$, it is differentiable at every $\zeta \in\left[M_{\beta},+\infty\right) \backslash L(t)$, with $|L(t)|=0$ (here by $|\cdot|$, we denote the Lebesgue measure on $\mathbb{R}$ ). We define

$$
\xi_{0}(t, \zeta) \stackrel{\mathrm{df}}{=} \begin{cases}\frac{\mathrm{d}}{\mathrm{d} \zeta}\left(\frac{j(t, \zeta)}{\zeta^{p}}\right) & \text { if } \zeta \in\left[M_{\beta},+\infty\right) \backslash L(t), \\ 0 & \text { if } \zeta \in L(t) .\end{cases}
$$

For all $t \in T \backslash E$ and all $\zeta \in\left[M_{\beta},+\infty\right) \backslash L(t)$ we have that $\xi_{0}(t, \zeta) \in \partial\left(j(t, \zeta) / \zeta^{p}\right)$ and so

$$
\xi_{0}(t, \zeta) \geqslant \frac{\beta}{\zeta^{p+1}} .
$$


Let $\eta, \bar{\eta} \in\left[M_{\beta},+\infty\right), \eta \leqslant \bar{\eta}$. Integrating the above inequality over the interval $[\eta, \bar{\eta}]$, we obtain

$$
\frac{j(t, \bar{\eta})}{\bar{\eta}^{p}}-\frac{j(t, \eta)}{\eta^{p}} \geqslant \frac{\beta}{p}\left(\frac{1}{\eta^{p}}-\frac{1}{\bar{\eta}^{p}}\right)
$$

Let $\bar{\eta} \rightarrow+\infty$. Because of hypothesis $H(j)_{2}$ (iv) we obtain

$$
\frac{j(t, \eta)}{\eta^{p}} \leqslant-\frac{\beta}{p \eta^{p}}
$$

and thus

$$
j(t, \eta) \leqslant-\frac{\beta}{p} \quad \forall \eta \geqslant M_{\beta}
$$

Since $\beta>0$ was arbitrary, it follows that $j(t, \eta) \rightarrow-\infty$ as $\eta \rightarrow+\infty$ uniformly for almost all $t \in T$. In a similar fashion we show that $j(t, \eta) \rightarrow-\infty$ as $\eta \rightarrow-\infty$ uniformly for almost all $t \in T$. Therefore, $j(t, \zeta) \rightarrow-\infty$ as $|\zeta| \rightarrow+\infty$ uniformly for almost all $t \in T$.

Let $\varphi \in W_{\text {per }}^{1, p}(T) \rightarrow \mathbb{R}$ be defined by

$$
\varphi(x) \stackrel{\mathrm{df}}{=} \frac{1}{p}\left\|x^{\prime}\right\|_{p}^{p}-\int_{0}^{b} j(t, x(t)) \mathrm{d} t .
$$

We will show that $\varphi$ is coercive. For this purpose let us assume that this is not true. Then we can find a sequence $\left\{x_{n}\right\}_{n \geqslant 1} \subseteq W_{\text {per }}^{1, p}(T)$ such that $\left\|x_{n}\right\|_{1, p} \rightarrow+\infty$ and $\left|\varphi\left(x_{n}\right)\right| \leqslant M_{3}$ for all $n \geqslant 1$, with some $M_{3}>0$. Let $y_{n} \stackrel{\text { df }}{=}\left(x_{n} /\left\|x_{n}\right\|_{1,2}\right)$ for $n \geqslant 1$. By passing to a subsequence if necessary, we may assume that

$$
y_{n} \rightarrow y \quad \text { weakly in } W_{\text {per }}^{1, p}(T)
$$

and

$$
y_{n} \rightarrow y \quad \text { in } C(T) .
$$

We have

$$
\frac{\varphi\left(x_{n}\right)}{\left\|x_{n}\right\|_{1, p}^{p}}=\frac{1}{p}\left\|y_{n}^{\prime}\right\|_{p}^{p}-\int_{0}^{b} \frac{j\left(t, x_{n}(t)\right)}{\left\|x_{n}\right\|_{1, p}^{p}} \mathrm{~d} t \leqslant \frac{M_{3}}{\left\|x_{n}\right\|_{1, p}^{p}} .
$$

Let us fix any $\beta>0$ and let $M_{\beta}>0$ be as at the beginning of this proof. We have

$$
\int_{0}^{b} \frac{j\left(t, x_{n}(t)\right)}{\left\|x_{n}\right\|_{1, p}^{p}} \mathrm{~d} t=\int_{\left\{\left|x_{n}\right|<M_{\beta}\right\}} \frac{j\left(t, x_{n}(t)\right)}{\left\|x_{n}\right\|_{1, p}^{p}} \mathrm{~d} t+\int_{\left\{\left|x_{n}\right| \geqslant M_{\beta}\right\}} \frac{j\left(t, x_{n}(t)\right)}{\left\|x_{n}\right\|_{1, p}^{p}} \mathrm{~d} t .
$$

As in the proof of Theorem 3.1, from hypotheses $H(j)_{2}$ (ii), (iii) and the mean value theorem (see Lebourg [15]) for almost all $t \in T$ and all $\zeta \in \mathbb{R}$ we have

$$
|j(t, \zeta)| \leqslant a_{1}(t)+c_{1}(t)|\zeta|^{r}
$$

with $a_{1} \in L 1(T)_{+}$and $c_{1} \in L^{r^{\prime}}(T)_{+}$. So for some $\alpha_{2} \in L^{1}(T)_{+}$we have

$$
\begin{gathered}
\left|\int_{\left\{\left|x_{n}\right|<M_{\beta}\right\}} \frac{j\left(t, x_{n}(t)\right)}{\left\|x_{n}\right\|_{1, p}^{p}} \mathrm{~d} t\right| \leqslant \int_{0}^{b} \frac{a_{2}(t)}{\left\|x_{n}\right\|_{1, p}^{p}} \mathrm{~d} t \rightarrow 0, \\
\int_{\left\{\left|x_{n}\right| \geqslant M_{\beta}\right\}} \frac{j\left(t, x_{n}(t)\right)}{\left\|x_{n}\right\|_{1, p}^{p}} \mathrm{~d} t \rightarrow 0
\end{gathered}
$$


as $n \rightarrow \infty$ because of hypothesis $H(j)_{2}$ (iv). So we see that

$$
\int_{0}^{b} \frac{j\left(t, x_{n}(t)\right)}{\left\|x_{n}\right\|_{1, p}^{p}} \mathrm{~d} t \rightarrow 0 \quad \text { as } n \rightarrow+\infty .
$$

Passing to the limit as $n \rightarrow+\infty$ in (3.5) and using this convergence, we obtain

$$
\left\|y^{\prime}\right\|_{p}=0, \quad \text { i.e. } y \equiv \eta \in \mathbb{R} \text {. }
$$

If $\eta=0$, then we have $\left\|y_{n}^{\prime}\right\|_{p} \rightarrow 0$, hence $y_{n}^{\prime} \rightarrow 0$ in $L^{p}(T)$ and so $y_{n} \rightarrow 0$ in $W_{\text {per }}^{1, p}(T)$, a contradiction since $\left\|y_{n}\right\|_{1, p}=1$ for all $n \geqslant 1$. Therefore, $\eta \neq 0$. This means that for all $t \in T$ we have $\left|x_{n}(t)\right| \rightarrow+\infty$ as $n \rightarrow+\infty$. We claim that this convergence is uniform in $t \in T$. Indeed let $\delta>0$ be such that $\delta<|\eta|$ (recall that $y \equiv \eta \neq 0$ ). Since $y_{n} \rightarrow \eta$ in $C(T)$, we can find $n_{0} \geqslant 1$ such that

$$
\left|y_{n}(t)-\eta\right|<\delta \quad \forall n \geqslant n_{0}, \quad \forall t \in T
$$

and hence

$$
\left|y_{n}(t)\right| \geqslant|\eta|-\delta=\delta_{1}>0 .
$$

Since by hypothesis $\left\|x_{n}\right\|_{1, p} \rightarrow+\infty$, for a given $\beta_{1}>0$, we can find $n_{1} \geqslant 1$ such that

$$
\left\|x_{n}\right\|_{1, p} \geqslant \beta_{1}>0 \quad \forall n \geqslant n_{1} .
$$

If $n_{2} \stackrel{\mathrm{df}}{=} \max \left\{n_{0}, n_{1}\right\}$, then for all $n \geqslant n_{2}$ and all $t \in T$ we have that

$$
\frac{\left|x_{n}(t)\right|}{\beta_{1}} \geqslant \frac{\left|x_{n}(t)\right|}{\left\|x_{n}\right\|_{1, p}}=\left|y_{n}(t)\right| \geqslant \delta_{1}>0
$$

and so

$$
\left|x_{n}(t)\right| \geqslant \beta_{1} \delta_{1} .
$$

Because $\beta_{1}>0$ was arbitrary and $\delta_{1}>0$, we conclude that $\left|x_{n}(t)\right| \rightarrow+\infty$ as $n \rightarrow+\infty$, uniformly in $t \in T$. But for a given $\beta_{2}>0$, we can find $n_{3} \geqslant 1$ such that

$$
j\left(t, x_{n}(t)\right) \leqslant-\beta_{2} \quad \forall n \geqslant n_{3} \text { and a.a. } t \in T
$$

(recall that $j(t, \zeta) \rightarrow-\infty$ as $|\zeta| \rightarrow+\infty$ uniformly for almost all $t \in T$ ). Then from the choice of the sequence $\left\{x_{n}\right\}_{n \geqslant 1} \subseteq W_{\text {per }}^{1, p}(T)$ for all $n \geqslant n_{3}$ we have

$$
\varphi\left(x_{n}\right) \leqslant M_{3} \quad \forall n \geqslant 1,
$$

so, in particular,

$$
-\int_{0}^{b} j\left(t, x_{n}(t)\right) \mathrm{d} t \leqslant M_{3} \quad \forall n \geqslant 1
$$

and thus

$$
\beta_{2} b \leqslant M_{3} .
$$

Recall that $\beta_{2}>0$ was arbitrary. So let $\beta_{2} \rightarrow+\infty$ to obtain a contradiction. Thus $\varphi$ is coercive. 
In particular, $\varphi$ is bounded below and satisfies the non-smooth Palais-Smale condition (see the proof of Theorem 3.1).

As in the proof of Theorem 3.1, we consider the direct sum decomposition

$$
W_{\text {per }}^{1, p}(T)=\mathbb{R} \oplus V \quad \text { with } V \stackrel{\text { df }}{=}\left\{v \in W_{\text {per }}^{1, p}(T): \int_{0}^{b} v(t) \mathrm{d} t=0\right\} .
$$

Using hypothesis $H(j)_{2}$ (vi) as in the proof of Theorem 3.1, we can find $\rho_{2}>0$ such that $\varphi(\eta) \leqslant 0$ for all $\eta \in \mathbb{R}$ with $|\eta| \leqslant \rho_{2}$ and $\varphi(v) \geqslant 0$ for all $v \in V$ with $\|v\|_{1, p} \leqslant \rho_{2}$. Thus we can apply Theorem 2.2 and obtain two distinct non-trivial critical points of $\varphi$, about which as before we can show that they are distinct non-trivial solutions of (HVI).

For semilinear problems (i.e. with $p=2$ ), exploiting the orthogonality relations, we can allow near the origin interaction with higher parts of the spectrum of minus the one-dimensional Laplacian with periodic boundary conditions (resonance at the origin). So we consider the following particular version of problem (HVI):

$$
\left.\begin{array}{c}
-x^{\prime \prime}(t) \in \partial j(t, x(t)) \quad \text { for almost all } t \in T=[0, b], \\
x(0)=x(b), \quad x^{\prime}(0)=x^{\prime}(b) .
\end{array}\right\}
$$

We impose the following conditions on the non-smooth potential $j(t, \zeta)$.

$\left(H(j)_{3}\right) . j: T \times \mathbb{R} \mapsto \mathbb{R}$ is a functional such that

(i) for all $\zeta \in \mathbb{R}$, the function $T \ni t \mapsto j(t, \zeta) \in \mathbb{R}$ is measurable;

(ii) for almost all $t \in T$, the function $\mathbb{R} \ni \zeta \mapsto j(t, \zeta) \in \mathbb{R}$ is locally Lipschitz and $j(t, 0)=0$

(iii) for almost all $t \in T$, all $\zeta \in \mathbb{R}$ and all $u \in \partial j(t, \zeta)$ we have

$$
|u| \leqslant a(t)+c(t)|\zeta|,
$$

with $a, c \in L^{2}(T)_{+}$;

(iv) $\lim \sup _{|b| \rightarrow+\infty}\left(j(t, \zeta) / \zeta^{2}\right) \leqslant h(t)$ uniformly for almost all $t \in T$ with $h \in L^{1}(T)$ such that $\int_{0}^{b} h(t) \mathrm{d} t<0 ;$ and

(v) there exist $\rho_{1}>0$ and $0<\mu<\lambda_{k+1}$, such that for almost all $t \in T$ and all $|\zeta| \leqslant \rho_{1}$ we have

$$
\lambda_{k} \zeta^{2} \leqslant 2 j(t, \zeta) \leqslant \mu \zeta^{2}
$$

(recall that $\lambda_{k}=(k \omega)^{2}$, with $\omega=(2 \pi / b)$ for $\left.k \geqslant 0\right)$.

Remark 3.4. Consider the potential function

$$
j(t, \zeta) \stackrel{\text { df }}{=} \begin{cases}\mathrm{e}^{\zeta}-\zeta^{2}+\mu+1-\frac{1}{\mathrm{e}} & \text { if } \zeta<-1, \\ \mu \zeta^{6} & \text { if }|\zeta| \leqslant 1, \\ h(t) \zeta^{2}-\zeta \ln \zeta^{2}+\mu-h(t) & \text { if } \zeta>1 .\end{cases}
$$


If $\lambda_{k} \leqslant \mu \leqslant \lambda_{k+1}, \rho_{1}=(1 / \sqrt[4]{2}), h \in L 1(T), h(t) \leqslant 0$, for almost all $t \in T$ with strict inequality on a set of positive measure, then $j(t, \zeta)$ satisfies hypotheses $H(j)_{3}$.

Theorem 3.5. If hypotheses $H(j)_{3}$ hold, then problem $\left(H V I^{\prime}\right)$ has at least two distinct non-trivial solutions.

Proof. Let $\varphi: W_{\text {per }}^{1,2}(T) \rightarrow \mathbb{R}$ be defined by

$$
\varphi(x) \stackrel{\mathrm{df}}{=} \frac{1}{2}\left\|x^{\prime}\right\|_{2}^{2}-\int_{0}^{b} j(t, x(t)) \mathrm{d} t .
$$

Again we have that $\varphi$ is locally Lipschitz. We claim that $\varphi$ is coercive. As before we argue by contradiction. Suppose that $\varphi$ is not coercive. We can find a sequence $\left\{x_{n}\right\}_{n \geqslant 1} \subseteq W_{\text {per }}^{1,2}(T)$ and $M_{4}$ such that $\left\|x_{n}\right\|_{1,2} \rightarrow+\infty$ and $\left|\varphi\left(x_{n}\right)\right| \leqslant M_{4}$ for all $n \geqslant 1$. Let $y_{n} \stackrel{\text { df }}{=}\left(x_{n} /\left\|x_{n}\right\|_{1,2}\right)$ for $n \geqslant 1$. We may assume that

$$
\begin{array}{ll}
y_{n} \rightarrow y & \text { weakly in } W_{\text {per }}^{1,2}(T), \\
y_{n} \rightarrow y \quad \text { in } C(T) .
\end{array}
$$

We have that

$$
\frac{1}{2}\left\|y_{n}^{\prime}\right\|_{2}^{2}-\int_{0}^{b} \frac{j\left(t, x_{n}(t)\right)}{\left\|x_{n}\right\|_{1,2}^{2}} \mathrm{~d} t \leqslant \frac{M_{4}}{\left\|x_{n}\right\|_{1,2}^{2}} .
$$

Recall that by virtue of hypothesis $H(j)_{3}$ (iii) and the Lebourg mean value theorem (see Lebourg [15]), for almost all $t \in T$ and all $\zeta \in \mathbb{R}$ we have that

$$
|j(t, \zeta)| \leqslant a_{1}(t)+c_{1}(t)|\zeta|^{2}
$$

with $a_{1} \in L^{1}(T)_{+}$and $c_{1} \in L^{2}(T)_{+}$. So

$$
\frac{\left|j\left(t, x_{n}(t)\right)\right|}{\left\|x_{n}\right\|_{1,2}^{2}} \leqslant \frac{a_{1}(t)}{\left\|x_{n}\right\|_{1,2}^{2}}+c_{1}(t)\left|y_{n}(t)\right|^{2} .
$$

By the Dunford-Pettis Theorem, we may assume that

$$
\frac{j\left(\cdot, x_{n}(\cdot)\right)}{\left\|x_{n}\right\|_{1,2}^{2}} \rightarrow \gamma(\cdot) \quad \text { weakly in } L^{1}(T) .
$$

For all $t \in\{\tau \in T: y(\tau) \neq 0\}$ we have that $\left|x_{n}(t)\right| \rightarrow+\infty$ as $n \rightarrow+\infty$. Let $\varepsilon>0$ and let us introduce the set

$$
C_{n}(\varepsilon) \stackrel{\mathrm{df}}{=}\left\{t \in T: x_{n}(t) \neq 0 \text { and } \frac{j\left(t, x_{n}(t)\right)}{\left|x_{n}(t)\right|^{2}} \leqslant h(t)+\varepsilon\right\} .
$$

We set $\chi_{n}(t)=\chi_{C_{n}}(t)$. We have $\chi_{n}(t) \rightarrow 1$ almost everywhere on $\{y \neq 0\}$ (see hypothesis $H(j)_{3}($ iv)). Also for all $n \geqslant 1$ and almost all $t \in T$ we have that

$$
\frac{j\left(t, x_{n}(t)\right)}{\left\|x_{n}\right\|_{1,2}^{2}} \chi_{n}(t)=\frac{j\left(t, x_{n}(t)\right)}{\left|x_{n}(t)\right|^{2}}\left|y_{n}(t)\right|^{2} \chi_{n}(t) \leqslant(h(t)+\varepsilon)\left|y_{n}(t)\right|^{2} \chi_{n}(t) .
$$


Passing to the weak limit in $L^{1}(\{y \neq 0\})$ and using [11, Proposition 7.3.9, p. 694], we obtain

$$
\gamma(t) \leqslant(h(t)+\varepsilon)|y(t)|^{2} \quad \text { a.e. on }\{y \neq 0\} .
$$

As $\varepsilon>0$ was arbitrary, we also have

$$
\gamma(t) \leqslant h(t)|y(t)|^{2} \quad \text { a.e. on }\{y \neq 0\} .
$$

On the other hand, it is clear from (3.8) that

$$
\gamma(t)=0 \quad \text { for a.a. } t \in\{y=0\} .
$$

So, finally,

$$
\gamma(t) \leqslant h(t)|y(t)|^{2} \quad \text { a.e. on } T
$$

and so

$$
\gamma(t)=\bar{h}(t)|y(t)|^{2} \quad \text { a.e. on } T,
$$

with $\bar{h} \in L^{1}(t), \bar{h}(t) \leqslant h(t)$ for almost all $t \in T$. Then, from hypothesis $H(j)_{3}$ (iv) and by passing to the limit in (3.6) as $n \rightarrow+\infty$, we obtain

$$
\frac{1}{2}\left\|y^{\prime}\right\|_{2}^{2} \leqslant \int_{0}^{b} \bar{h}(t)|y(t)|^{2} \mathrm{~d} t \leqslant\|y\|_{C(T)}^{2} \int_{0}^{b} \bar{h}(t) \mathrm{d} t \leqslant 0
$$

and so

$$
y \equiv \eta \in \mathbb{R} .
$$

If $\eta=0$, then as before we have that $y_{n} \rightarrow 0$ in $W_{\text {per }}^{1,2}(T)$, a contradiction, since $\left\|y_{n}\right\|_{1,2}=1$ for all $n \geqslant 1$. Hence $\eta \neq 0$ and we have

$$
0 \leqslant \int_{0}^{b} \bar{h}(t) \eta^{2} \mathrm{~d} t \leqslant \eta^{2} \int_{0}^{b} h(t) \mathrm{d} t<0
$$

a contradiction. This proves the coercivity of $\varphi$, which in turn implies that $\varphi$ is bounded below and satisfies the non-smooth Palais-Smale condition (see the proof of Theorem 3.1).

Next let

$$
W_{k} \stackrel{\text { df }}{=}\left\{x \in W_{\text {per }}^{1,2}(T): x(t)=\sum_{i=0}^{k}\left(a_{i} \sin \frac{2 \pi i}{b} t+b_{i} \cos \frac{2 \pi i}{b} t\right), a_{i}, b_{i} \in \mathbb{R}\right\} .
$$

Let $\rho_{1}>0$ be as in hypothesis $H(j)_{3}(\mathrm{v})$. From the compactness of the embedding $W_{\text {per }}^{1,2}(T) \subseteq C(T)$, we know that we can find $\rho_{2}>0$ such that for all $v \in W_{k}$, with $\|v\|_{1,2} \leqslant \rho_{2}$, we have

$$
\|v\|_{C(T)}=\max _{t \in T}|v(t)| \leqslant \rho_{1} .
$$


Using hypothesis $H(j)_{3}(\mathrm{v})$, for all $v \in W_{k}$, we obtain

$$
\varphi(v)=\frac{1}{2}\left\|v^{\prime}\right\|_{2}^{2}-\int_{0}^{b} j(t, v(t)) \mathrm{d} t \leqslant \frac{1}{2}\left\|v^{\prime}\right\|_{2}^{2}-\frac{1}{2} \lambda_{k}\|v\|_{2}^{2} \leqslant 0
$$

(the last inequality follows from the fact that $\left(\left\|v^{\prime}\right\|_{2}^{2} /\|v\|_{2}^{2}\right) \leqslant \lambda_{k}$ for all $v \in W_{k}$ (see Mawhin and Willem [18, p. 156])).

Let $w \in W_{k}^{\perp}$. Again we can find $0<\rho_{3} \leqslant \rho_{2}$ such that if $\|w\|_{1,2} \leqslant \rho_{3}$, then

$$
\|w\|_{C(T)}=\max _{t \in T}|w(t)| \leqslant \rho_{1} .
$$

So we have

$$
\begin{aligned}
\varphi(w) & =\frac{1}{2}\left\|w^{\prime}\right\|_{2}^{2}-\int_{0}^{b} j(t, w(t)) \mathrm{d} t \\
& =\frac{1}{2}\left\|w^{\prime}\right\|_{2}^{2}-\int_{\left\{|w| \leqslant \rho_{1}\right\}} j(t, w(t)) \mathrm{d} t-\int_{\left\{|w|>\rho_{1}\right\}} j(t, w(t)) \mathrm{d} t .
\end{aligned}
$$

From (3.7), for a given $s>2$, we can find $c_{2} \in L^{1}(T)_{+}$such that for almost all $t \in T$ and all $|\zeta| \geqslant \rho_{1}$ we have

$$
j(t, \zeta) \leqslant c_{2}(t)|\zeta|^{s}
$$

Thus from hypothesis $H(j)_{3}$ (v) we have

$$
\varphi(w) \geqslant \frac{1}{2}\left\|w^{\prime}\right\|_{2}^{2}-\frac{1}{2} \mu\|w\|_{2}^{2}-\beta_{3}\|w\|_{1,2}^{s},
$$

for some $\beta_{3}>0$. As $\left(\left\|w^{\prime}\right\|_{2}^{2} /\|w\|_{2}^{2}\right) \geqslant \lambda_{k+1}>\mu$ for all $w \in W_{k}^{\perp} \backslash\{0\}$, so

$$
\varphi(w) \geqslant \beta_{4}\|w\|_{1,2}^{2}-\beta_{3}\|w\|_{1,2}^{s},
$$

for some $\beta_{4}>0$. Because $s>2$, we can find $\rho_{4}<\min \left\{\rho_{3}, 1\right\}$ such that

$$
\varphi(w) \geqslant 0 \quad \forall w \in W_{k}^{\perp} \quad \text { with }\|w\|_{1,2} \leqslant \rho_{4} .
$$

Therefore, we can apply Theorem 2.2 and obtain two distinct non-trivial critical points of $\varphi$. We know (see the proof of Theorem 3.1) that these are two distinct non-trivial solutions of $\left(\mathrm{HVI}^{\prime}\right)$.

Remark 3.6. As we already mentioned in $\S 1$, for 'smooth' problems (i.e. $j(t, \cdot) \in$ $C^{1}(\mathbb{R})$ ) only Del Pino, Manasevich and Murua [7] addressed the problem of multiple periodic solutions. However, their approach is different and uses the Fučik spectrum of the scalar $p$-Laplacian. Moreover, they assume that the right-hand side nonlinearity $f(t, x)(=\partial j(t, x))$ is continuous in both variables $(t, x) \in T \times \mathbb{R}$.

When $p=1$, note that the map $x \rightarrow \psi_{p}(x)=|x|^{p-2} x$ is no longer a homeomorphism on $\mathbb{R}$ and the resulting differential operator is highly singular and to our knowledge no work exists in the literature for this case. One can check that in $[\mathbf{6 - 1 0}, \mathbf{1 3}, \mathbf{1 6}]$, which deal with the $p$-Laplacian (or extensions of it), it is assumed that $p>1$. 


\section{An eigenvalue problem}

In this section we consider the following nonlinear periodic eigenvalue problem:

$$
\left.\begin{array}{c}
-\left(\left|x^{\prime}(t)\right|^{p-2} x^{\prime}(t)\right)^{\prime}-\lambda\left(|x(t)|^{p-2} x(t)\right) \in \partial j(t, x(t)) \quad \text { for a.a. } t \in T=[0, b], \\
x(0)=x(b), \quad x^{\prime}(0)=x^{\prime}(b) .
\end{array}\right\}
$$

We shall show that problem $(\mathrm{EP})_{1}$ has at least three distinct solutions as $\lambda \rightarrow 0^{-}$(a problem near resonance). This way we give a partial extension to the non-smooth case and the $p$-Laplacian of a semilinear smooth result of Mawhin and Schmitt [17, Theorem 4].

Our hypotheses on $j(t, \zeta)$ are as follows.

$\left(H(j)_{4}\right) \cdot j: T \times \mathbb{R} \mapsto \mathbb{R}$ is a functional such that

(i) for all $\zeta \in \mathbb{R}$, the function $T \ni t \mapsto j(t, \zeta) \in \mathbb{R}$ is measurable and $j(\cdot, 0) \in L^{1}(T)$;

(ii) for almost all $t \in T$, the function $\mathbb{R} \ni \zeta \mapsto j(t, \zeta) \in \mathbb{R}$ is locally Lipschitz;

(iii) for almost all $t \in T$, all $\zeta \in \mathbb{R}$ and all $u \in \partial j(t, \zeta)$ we have

$$
|u| \leqslant a(t)+c(t)|\zeta|^{r-1},
$$

with $a, c \in L^{r^{\prime}}(T)$, where $1 \leqslant r<p$ and $(1 / r)+\left(1 / r^{\prime}\right)=1$;

(iv) $\lim _{|\zeta| \rightarrow+\infty}(\zeta u-p j(t, \zeta))=-\infty$ uniformly for almost all $t \in T$ and all $u \in \partial j(t, \zeta)$; and

(v) there exist $M>0$ and $\bar{\xi}>0$ such that for almost all $t \in T$, all $|\zeta| \geqslant M$ and all $u \in \partial j(t, \zeta)$ we have $\zeta u \geqslant \bar{\xi}$.

Theorem 4.1. If hypotheses $H(j)_{4}$ hold, then there exists $\hat{\lambda}>0$ such that for all $\lambda \in[-\hat{\lambda}, 0)$ problem $(E P)_{1}$ has at least three distinct solutions.

Proof. For $\lambda<0$, let $\varphi_{\lambda}: W_{\text {per }}^{1, p}(T) \rightarrow \mathbb{R}$ be the locally Lipschitz functional defined by

$$
\varphi_{\lambda}(x) \stackrel{\mathrm{df}}{=} \frac{1}{p}\left\|x^{\prime}\right\|_{p}^{p}-\frac{\lambda}{p}\|x\|_{p}^{p}-\int_{0}^{b} j(t, x(t)) \mathrm{d} t .
$$

From the mean value theorem of Lebourg [15] and hypotheses $H(j)_{4}$ (ii), (iii), for almost all $t \in T$ and all $\zeta \in \mathbb{R}$, we have that

$$
|j(t, \zeta)| \leqslant a_{1}(t)+c_{1}(t)|\zeta|^{r},
$$

with $a_{1} \in L^{1}(T)_{+}$, and $c_{1} \in L^{r^{\prime}}(T)_{+}$. So

$$
\varphi_{\lambda}(x) \geqslant c_{2}(\lambda)\|x\|_{1, p}^{p}-c_{3}\|x\|_{1, p}^{r}-c_{4},
$$

for some $c_{2}(\lambda), c_{3}, c_{4}>0$ (recall that $\lambda<0$ ). As $r<p$ so $\varphi_{\lambda}$ is coercive. So it is bounded below and satisfies the non-smooth Palais-Smale condition. 
Now consider the direct sum decomposition

$$
W_{\text {per }}^{1, p}(T)=\mathbb{R} \oplus V \quad \text { with } V \stackrel{\text { df }}{=}\left\{v \in W_{\text {per }}^{1, p}(T): \int_{0}^{b} v(t) \mathrm{d} t=0\right\} .
$$

Using the Poincaré-Wirtinger inequality (see Mawhin and Willem [18, p. 8]), for all $v \in V$ we have that

$$
\begin{aligned}
\varphi_{\lambda}(v) & =\frac{1}{p}\left\|v^{\prime}\right\|_{p}^{p}-\frac{\lambda}{p}\|v\|_{p}^{p}-\int_{0}^{b} j(t, v(t)) \mathrm{d} t \\
& \geqslant \frac{1}{p}\left(1-\lambda b^{p}\right)\left\|v^{\prime}\right\|_{p}^{p}-c_{5}-c_{6}\left\|v^{\prime}\right\|_{p}^{r},
\end{aligned}
$$

for some $c_{5}, c_{6}>0$ and thus $\varphi_{\lambda}$ is coercive on $V$ uniformly for $\lambda<0$. So we can find $m>0$ such that

$$
\varphi_{\lambda}(v) \geqslant-m \quad \forall \lambda<0, \quad \forall v \in V .
$$

Consider the following two disjoint open sets in $W_{\text {per }}^{1, p}(T)$ :

$$
\begin{aligned}
& U^{+} \stackrel{\text { df }}{=}\left\{x \in W_{\text {per }}^{1, p}: \int_{0}^{b} x(t) \mathrm{d} t>0\right\}, \\
& U^{-} \stackrel{\text { df }}{=}\left\{x \in W_{\text {per }}^{1, p}: \int_{0}^{b} x(t) \mathrm{d} t<0\right\} .
\end{aligned}
$$

Next we will show that there exist $\xi_{0} \in \mathbb{R}$ and $\hat{\lambda}<0$ such that

$$
\varphi_{\lambda}\left( \pm \xi_{0}\right)<-m \quad \forall \lambda \in[\hat{\lambda}, 0) .
$$

First, for a given $\xi \in \mathbb{R}$ we have

$$
\varphi_{\lambda}(\xi)=-\frac{\lambda}{p}|\xi|^{p} b-\int_{0}^{b} j(t, \xi) \mathrm{d} t .
$$

By virtue of hypothesis $H(j)_{4}$ (iv), for a given $\beta>(\mathrm{pm} / \mathrm{b})$, we can find $\xi_{0}>\beta$ such that for almost all $t \in T$, all $|\zeta| \geqslant \xi_{0}$ and all $u \in \partial j(t, \zeta)$ we have

$$
\zeta u-p j(t, \zeta) \leqslant-\beta .
$$

Also, by hypothesis $H(j)_{4}(\mathrm{v})$, we can find $\hat{\lambda}<0$ such that for almost all $t \in T$ and all $u \in \partial j\left(t, \xi_{0}\right)$ we have

$$
-\hat{\lambda} \xi_{0}^{p} \leqslant \xi_{0} u
$$

So using (4.2) and (4.3), for all $u \in \partial j\left(t, \xi_{0}\right)$ we have

$$
\begin{aligned}
\varphi_{\hat{\lambda}}\left(\xi_{0}\right) & =-\frac{\hat{\lambda}}{p} \xi_{0}^{p} b-\int_{0}^{b} j\left(t, \xi_{0}\right) \mathrm{d} t \\
& \leqslant \frac{1}{p} \int_{0}^{b}\left(\xi_{0} u-p j\left(t, \xi_{0}\right)\right) \mathrm{d} t<-\frac{\beta}{p} b<-m .
\end{aligned}
$$




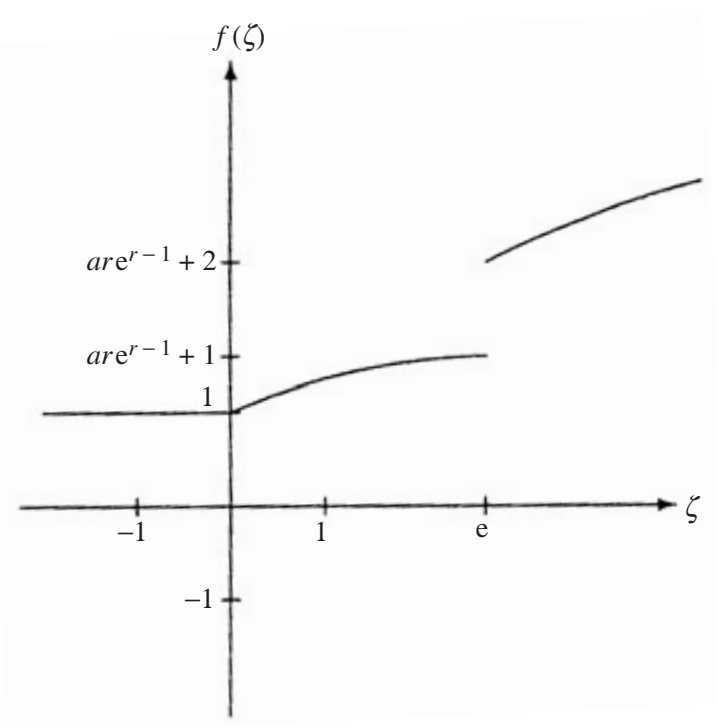

Figure 1.

Therefore, if $\lambda \in[\hat{\lambda}, 0)$, we have that $\varphi_{\lambda}\left(\xi_{0}\right)<-m$. In a similar fashion we can show that for all $\lambda \in[\hat{\lambda}, 0), \varphi_{\lambda}\left(-\xi_{0}\right)<-m$. So we have proved (4.1).

Next let

$$
\vartheta_{ \pm}^{\lambda} \stackrel{\text { df }}{=} \inf _{U^{ \pm}} \varphi_{\lambda}
$$

Note that by virtue of the coercivity of $\varphi_{\lambda}$, the numbers $\vartheta_{ \pm}^{\lambda}$ are finite and because of the statement proved above, we see that if $\lambda \in[\hat{\lambda}, 0)$, then $\vartheta_{ \pm}^{\lambda}<-m$. Moreover, since $\varphi_{\lambda}$ satisfies the non-smooth Palais-Smale condition, we can find $x_{ \pm}^{\lambda} \in \bar{U}^{ \pm}$such that $\varphi_{\lambda}\left(x_{ \pm}^{\lambda}\right)=\vartheta_{ \pm}^{\lambda}$ for $\lambda \in[\hat{\lambda}, 0)$. If $x_{ \pm}^{\lambda} \in \operatorname{bd} \bar{U}^{ \pm}=V$, then $\vartheta_{ \pm}^{\lambda} \geqslant-m$, a contradiction since $\lambda \in[\hat{\lambda}, 0)$. So $x_{ \pm}^{\lambda} \in U^{ \pm}$and so $x_{ \pm}^{\lambda}$ are distinct local minima of $\varphi_{\lambda}$, hence $0 \in \partial \varphi_{\lambda}\left(x_{ \pm}^{\lambda}\right)$ for $\lambda \in[\hat{\lambda}, 0)$.

Because $\left.\varphi_{\lambda}\right|_{V} \geqslant-m>\varphi_{\lambda}\left( \pm \xi_{0}\right)$, we can apply Theorem 2.1 and obtain $y_{\lambda} \in W_{\text {per }}^{1, p}(T)$ such that $0 \in \partial \varphi_{\lambda}\left(y_{\lambda}\right)$ and $\varphi_{\lambda}\left(y_{\lambda}\right) \geqslant-m>\varphi_{\lambda}\left(x_{ \pm}^{\lambda}\right)$, with $\lambda \in[\hat{\lambda}, 0)$. Hence $y_{\lambda} \neq x_{ \pm}^{\lambda}$ and as in the proof of Theorem 3.1, we can check that $x_{ \pm}^{\lambda}$ and $y_{\lambda}$ are solutions of $(\mathrm{EP})_{1}$ for all $\lambda \in[\hat{\lambda}, 0)$.

We will end this section with a simple example illustrating the applicability of our result. The example is in the spirit of those of Panagiotopoulos [19], analysed there in the context of mechanical systems.

First, let us consider the following function $f: \mathbb{R} \mapsto \mathbb{R}$ (we drop $t$-dependence for simplicity)

$$
f(\zeta) \stackrel{\mathrm{df}}{=} \begin{cases}1 & \text { if } \zeta<0, \\ 1+r a \zeta^{r-1} & \text { if } 0 \leqslant \zeta<\mathrm{e}, \\ 1+r a \zeta^{r-1}+\ln \zeta & \text { if } \mathrm{e} \leqslant \zeta,\end{cases}
$$

where $a>0$ and $1 \leqslant r \leqslant p$ (see figure 1$)$. 


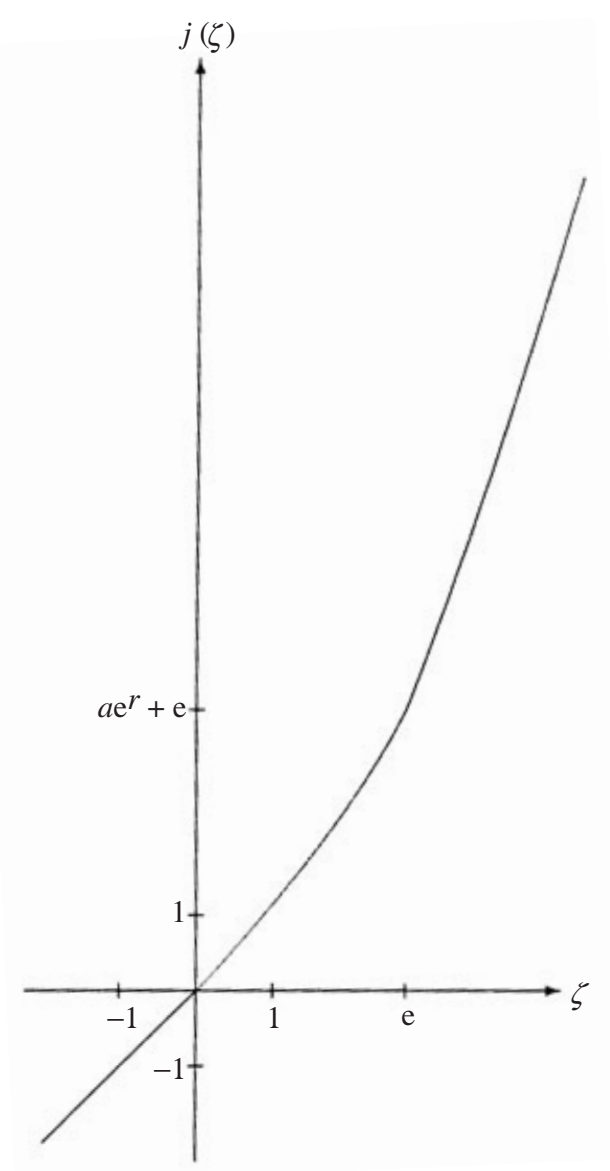

Figure 2.

Let $j: \mathbb{R} \mapsto \mathbb{R}$ be defined by $j(\zeta) \stackrel{\mathrm{df}}{=} \int_{0}^{\zeta} f(\xi) \mathrm{d} \xi$ (see figure 2 ). Then

$$
j(\zeta) \stackrel{\text { df }}{=} \begin{cases}\zeta & \text { if } \zeta \leqslant 0 \\ a \zeta^{r}+\max \{\zeta, \zeta \ln \zeta\} & \text { if } \zeta>0\end{cases}
$$

Note that $j$ is not differentiable at e. At this point $f$ exhibits a jump discontinuity. Let us define the multifunction $\hat{f}: \mathbb{R} \mapsto 2^{\mathbb{R}}$, by 'filling in the gaps' at the discontinuity point of $f$ (see figure 3). From Clarke [5, p. 34], we know that $j$ is locally Lipschitz and $\partial j(\zeta)=\hat{f}(\zeta)$ for all $\zeta \in \mathbb{R}$.

If we are given the problem

$$
\begin{gathered}
-\left(\left|x^{\prime}(t)\right|^{p-2} x^{\prime}(t)\right)^{\prime}-\lambda\left(|x(t)|^{p-2} x(t)\right)=f(x(t)) \quad \text { for a.a. } t \in T=[0, b], \\
x(0)=x(b), \quad x^{\prime}(0)=x^{\prime}(b),
\end{gathered}
$$




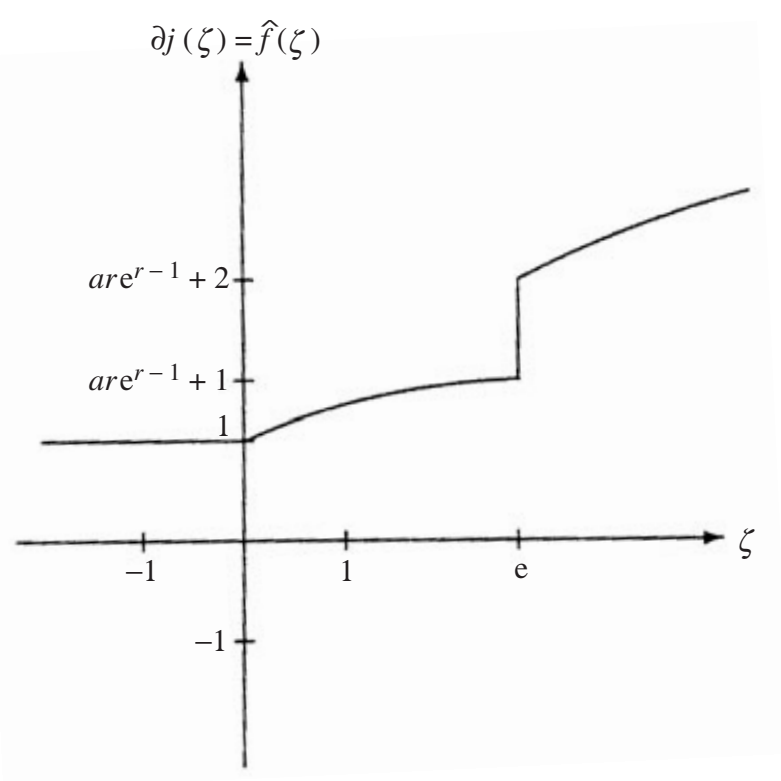

Figure 3.

which need not have a solution (due to the discontinuity of $f$ ), we replace it with the multivalued problem

$$
\left.\begin{array}{c}
-\left(\left|x^{\prime}(t)\right|^{p-2} x^{\prime}(t)\right)^{\prime}-\lambda\left(|x(t)|^{p-2} x(t)\right) \in \partial j(x(t)) \quad \text { for a.a. } t \in T=[0, b], \\
x(0)=x(b), \quad x^{\prime}(0)=x^{\prime}(b) .
\end{array}\right\}
$$

It is easy to verify that $j$ satisfies hypotheses $H(j)_{4}$ and so, by Theorem 4.1 , there exists $\hat{\lambda}>0$ such that for all $\lambda \in[-\hat{\lambda}, 0)$, problem (4.5) admits at least three distinct solutions.

Acknowledgements. L.G. was partly supported by KBN grants nos 7 T07A 04718 and 2 P03A 004 19. The authors thank the referee and the editor Dr Bryan Rynne for remarks that helped improve the paper.

\section{References}

1. R. AdAms, Sobolev spaces (Academic, 1975).

2. S. AdLy AND D. Goeleven, Periodic solutions for a class of hemivariational inequalities, Commun. Appl. Nonlin. Analysis 2 (1995), 45-57.

3. H. Brezis and L. Nirenberg, Remarks on finding critical points, Commun. Pure Appl. Math. 44 (1991), 939-963.

4. K. C. CHANG, Variational methods for nondifferentiable functionals and their applications to partial differential equations, J. Math. Analysis Applic. 80 (1981), 102-129.

5. F. H. Clarke, Optimization and nonsmooth analysis (Wiley, 1983).

6. H. DANG AND S. F. Oppenheimer, Existence and uniqueness results for some nonlinear boundary value problems, J. Math. Analysis Applic. 198 (1996), 35-48.

7. M. Del Pino, R. Manasevich and A. Murua, Existence and multiplicity of solutions with prescribed period for a second order ODE, Nonlin. Analysis 18 (1992), 79-92. 
8. C. FABRY AND D. FAYYAD, Periodic solutions of second order differential equations with a $p$-Laplacian and asymmetric nonlinearities, Rend. Istit. Mat. Univ. Trieste 24 (1992), 207-227.

9. L. GASiński AND N. S. PAPAgEORgIOU, A multiplicity result for nonlinear second order periodic equations with nonsmooth potential, Bull. Belgian Math. Soc. 9 (2002), 245-258.

10. Z. Guo, Boundary value problems of a class of quasilinear differential equations, Diff. Integ. Eqns 6 (1993), 705-719.

11. S. HU AND N. S. PAPAGEORGIOU, Handbook of multivalued analysis, vol. I, Theory (Kluwer, Dordrecht, 1997).

12. S. Hu And N. S. Papageorgiou, Handbook of multivalued analysis, vol. II, Applications (Kluwer, Dordrecht, 2000).

13. N. Kourogenis And N. S. PApageorgiou, Nonsmooth critical point theory and nonlinear elliptic equations at resonance, J. Aust. Math. Soc. A 69 (2000), 245-271.

14. N. Kourogenis, P. Kandilakis and N. S. Papageorgiou, Two nontrivial critical points for nonsmooth functionals and applications, Atti Seminario Matematico Modena, in press.

15. G. Lebourg, Valeur moyenne pour gradient généralisé, C. R. Acad. Sci. Paris Sér. I 281 (1975), 795-797.

16. J. MAWhin, Periodic solutions of systems with $p$-Laplacian-like operators, in Nonlinear Analysis and Applications to Differential Equations, Lisbon, 1997, Progress in Nonlinear Differential Equations and Applications, pp. 37-63 (Birkhauser, Boston, MA, 1998).

17. J. MAWHIN AND K. SCHMitT, Nonlinear eigenvalue problems with the parameter near resonance, Annls Polonici Math. 51 (1990), 241-248.

18. J. MAWHIN AND M. Willem, Critical point theory and Hamiltonian systems (Springer, 1989).

19. P. D. Panagiotopoulos, Hemivariational inequalities. Applications to mechanics and engineering (Springer, 1993). 